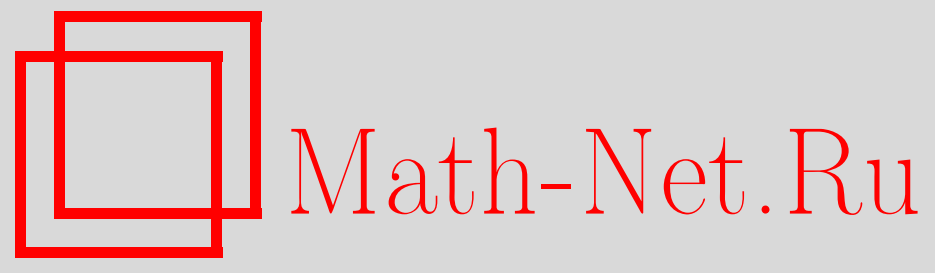

В. Г. Овчинников, $\mathrm{K}$ алгоритмам динамического программирования при предположениях монотонности, Вестн. Сам. гос. техн. ун-та. Сер. Физ.мат. науки, 2014, выпуск 1(), 186-191

DOI: https://doi.org/10.14498/vsgtu1257

Использование Общероссийского математического портала MathNet.Ru подразумевает, что вы прочитали и согласны с пользовательским соглашением

http://www . mathnet.ru/rus/agreement

Параметры загрузки:

IP: 54.198 .187 .58

26 апреля 2023 г., 04:33:23

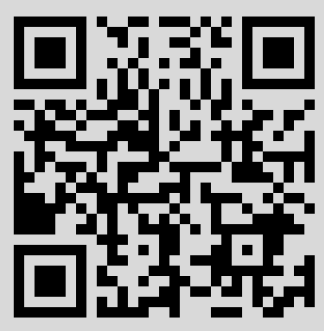


Вестн. Сам. гос. техн. ун-та. Сер. Физ.-мат. науки. 2014. № 1 (34). С. $186-191$

\title{
Информатика
}

\author{
УДК 519.7
}

\section{К АЛГОРИТМАМ ДИНАМИЧЕСКОГО ПРОГРАММИРОВАНИЯ ПРИ ПРЕДПОЛОЖЕНИЯХ МОНОТОННОСТИ}

\author{
В. Г. Овчинников \\ Самарский государственный технический университет, \\ Россия, 443100, Самара, ул. Молодогвардейская, 244.
}

\begin{abstract}
Формулируется задача дискретного оптимального управления, не рассматривавшаяся ранее и возникающая при проектировании нефтегазосборных сетей. Для этой задачи устанавливаются четыре теоремы, чтобы можно было иметь процесс, оптимальный процесс и оптимальное значение. Необходимые и достаточные условия для этого даются в теореме 1. При этих условиях по теореме 1 получаются интерваль достижимости, которые не пусты. Для каждого интервала выбирается сетка - подмножество его точек, где по произвольной точке интервала находится ближайшая точка слева. При помощи таких приближений определяются на сетках функиии Беллмана. С использованием функиий Беллмана в теореме 2 даётся процесс и оценивается отклонение его от оптимального процесса. В теореме 2 гарантируется, что процесс, который даётся там, оптимален в случае, когда интерваль достижимости и их сетки совпадают. В других случаях для получения оптималъного процесса исполъзуются теоремы 3 и теоремы 4. В теореме 3 устанавливается, что проиесс, который даётся в теореме 2, минимален в лексикографическом порядке, который вводится с использованием функиий Беллмана. В теореме 3 даётся процедура, которая строит, если возможно, в этом порядке следующий процесс, пропуская лишь процессы, которье неоптимальны. Оптимальный процесс и оптимальное значение находятся по теореме 4 исходя из процесса, который даётся в теореме 2, при помощи одного или нескольких вызовов процедуръ, которая даётся в теореме 3 .
\end{abstract}

Ключевые слова: динамическое программирование, функиии Беллмана, процедуры, алгоритмы.

1. Статья продолжает исследования применения динамического программирования при предположениях монотонности [1]. В ней в отличие от работ [1-3] устанавливаются утверждения (теоремы 1-4), обосновывающие отыскание оптимального процесса, для введения которого служит являющаяся случаем [3] следующая задача.

ISSN: 2310-7081 (online), 1991-8615 (print); doi: http://dx.doi.org/10.14498/vsgtu1257 (C) 2014 Самарский государственный технический университет.

Образец цитирования: В. Г. О в ч и н н и к о в, "K алгоритмам динамического программирования при предположениях монотонности" // Вестн. Сам. гос. техн. ун-та. Сер. Физ.мат. науки, 2014. № 1 (34). C. 186-191. doi: 10.14498/vsgtu1257.

Сведения об авторе: Валерий Гаврилович Овчинников, старший преподаватель, каф. разработки нефтяных и газовых месторождений.

E-mail address: ovchinnikov42@mail.ru 
ЗАДАчА. Пусть известно следующее:

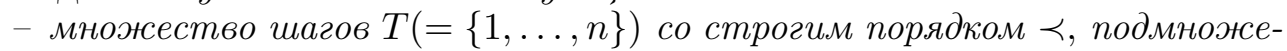
ством

$$
T_{0}=\{i \in T: \emptyset=\{j \in T: j \prec i\}\}(\neq T)
$$

начальных шагов, определением $\pi(i)$ как единственного шага в подмножестве предшествующих непосредственно $i$ шагов

$$
\{j \in T:(j \prec i) \wedge(\emptyset=\{g \in T: j \prec g \prec i\})\}\left(\forall i \in T \backslash T_{0}\right)
$$

и удовлетворяющим равенству $\emptyset=M \cap T_{0}$ подмножсеством последних шагов

$$
M=\{i \in T: \tau(i)=\emptyset\},
$$

где $\tau(i)=\left\{j \in T \backslash T_{0}: \pi(j)=i\right\}$;

- непустые интерваль $\left[a_{i} . . b_{i}\right]\left(=\left\{\alpha \in \mathbb{Z}: a_{i} \leqslant \alpha \leqslant b_{i}\right\}\right)$ множества иелых чисел $\mathbb{Z}(\forall i \in T)$;

- бункиия $\mathbf{s}$ со значениями

$$
s_{i}(\alpha, \beta) \in\left\{\gamma \in \mathbb{Z}: a_{i} \leqslant \gamma\right\}\left(\forall i \in T \backslash T_{0}\right)(\forall \alpha, \beta \in \mathbb{Z}),
$$

функиия $f$ с значениями $f_{i}(\alpha, \beta) \in \mathbb{R}$ (во множестве вещественных чисел) $\left(\forall i \in T \backslash T_{0}\right)(\forall \alpha, \beta \in \mathbb{Z})$ и функиия $U$ с конечными $\left(\left\|U_{i}(\alpha)\right\|<\infty\right)$ значениями

$$
U_{i}(\alpha) \subset \mathbb{Z}\left(\forall i \in T \backslash T_{0}\right)(\forall \alpha \in \mathbb{Z})
$$

при предположениях монотонности

$$
\begin{gathered}
(\alpha \leqslant \delta) \Rightarrow\left(f_{i}(\alpha, \beta) \leqslant f_{i}(\delta, \beta)\right) \wedge\left(s_{i}(\alpha, \beta) \leqslant s_{i}(\delta, \beta)\right) \wedge\left(\Gamma_{i}(\alpha, \beta) \supseteq \Gamma_{i}(\delta, \beta)\right) \\
\left(\forall i \in T \backslash T_{0}\right)(\forall \alpha, \beta, \delta \in \mathbb{Z}),
\end{gathered}
$$

где $\Gamma_{i}(\alpha, \beta)=\left\{\gamma \in U_{i}(\alpha): s_{i}(\alpha, \gamma) \leqslant \beta\right\}$

- обозначение $D$ множества называемых процессами пар $(x, u)$ функиии $x, u$ со значениями $x_{i} \in \mathbb{Z}(\forall i \in T), u_{i} \in \mathbb{Z}(\forall i \in T)$, удовлетворяющих ограничениям $x_{i}=u_{i}\left(\forall i \in T_{0}\right), u_{i} \in U_{i}\left(x_{\pi(i)}\right)\left(\forall i \in T \backslash T_{0}\right), x_{i}=$ $=s_{i}\left(x_{\pi(i)}, u_{i}\right)\left(\forall i \in T \backslash T_{0}\right), x_{i} \in\left[a_{i} . . b_{i}\right](\forall i \in T)$.

Требуется найти оптимальный процесс $\left(x^{*}, u^{*}\right)$ и оптимальное значение $r^{*}$ из условий

$$
\begin{gathered}
\left(x^{*}, u^{*}\right) \in D, \quad F\left(x^{*}, u^{*}\right)=r^{*} \\
r^{*}=\min _{(x, u) \in D} F(x, u), F(x, u)=\sum_{i \in T \backslash T_{0}} f_{i}\left(x_{\pi(i)}, u_{i}\right)(\forall(x, u) \in D) .
\end{gathered}
$$

ЗАмечАниЕ. В частном случае получающегося, когда $\mathbb{Z}$ заменяется на $\mathbb{R}$, варианта этой задачи существование оптимального процесса охарактеризовано в [1].

В общем случае с использованием

$$
T_{k}=\{i \in T: h(i)=k\}(\forall k \in[1 . . h(T)]),
$$


где

$$
h(i)=\|\{j \in T: j \prec i\}\|, \quad h(T)=\max _{i \in T} h(i)
$$

характеризацию существования оптимального процесса даёт следующая теорема.

ТЕорема 1. Для задачи следующие условия равносильны:

- оптимальный процесс существует;

- индукиией

$$
a_{i}^{0}=a_{i}\left(\forall i \in T_{0}\right), \quad a_{i}^{0}=\min _{\beta \in U_{i}\left(a_{\pi(i)}^{0}\right)} s_{i}\left(a_{\pi(i)}^{0}, \beta\right)\left(\forall i \in T_{k}\right)
$$

$($ вверх по $k)(\forall k \in[1 . . h(T)])$ определяются числа $a_{i}^{0} \in\left[a_{i} . . b_{i}\right](\forall i \in T)$.

При выполнении этих условий с помощъю равенств

$$
\left[a_{i}^{0} . . b_{i}^{0}\right]=\left[a_{i}^{0} . . b_{i}\right](\forall i \in M)
$$

индукиией

$$
\left[a_{i}^{0} . . b_{i}^{0}\right]=\left\{\alpha \in\left[a_{i}^{0} . . b_{i}\right]: \Gamma_{j}\left(\alpha, b_{j}^{0}\right) \neq \emptyset(\forall j \in \tau(i))\right\}\left(\forall i \in T_{k} \backslash M\right)
$$

$($ вниз по $k)(\forall k \in[0 . . h(T)-1])$ получаются множества достижимости $\left[a_{i}^{0} . . b_{i}^{0}\right](\forall i \in T)\left(\right.$ ср. [2]), имеюшие элементы $x_{i}(\forall(x, u) \in D)$.

2. Далее предполагается, что по теореме 1 найдены непустые интервалы $\left[a_{i}^{0} . . b_{i}^{0}\right](\forall i \in T)$, и, следовательно, для множеств $U_{i}^{0}(\alpha)=\Gamma_{i}\left(\alpha, b_{i}^{0}\right)$ выполнены условия

$U_{i}^{0}(\alpha) \neq \emptyset \quad$ и $\quad s_{i}(\alpha, \gamma) \in\left[a_{i}^{0} . . b_{i}^{0}\right]\left(\forall \gamma \in U_{i}^{0}(\alpha)\right)\left(\forall \alpha \in\left[a_{\pi(i)}^{0} . . b_{\pi(i)}^{0}\right]\right)\left(\forall i \in T \backslash T_{0}\right)$

Также предполагается следующее:

- $D^{0}=\left\{(x, u) \in D: u_{i}=a_{i}\left(\forall i \in T_{0}\right)\right\}$;

- $\left(\left\{a_{i}^{0}\right\} \subset Y_{i} \subseteq\left[a_{i}^{0} . . b_{i}^{0}\right]\right) \wedge\left(\left\|Y_{i}\right\| \leqslant c_{i}\right)$ (где $c_{i}$ - заданные границы мощностей) $(\forall i \in T)$;

$-\left(a_{i}^{0} \leqslant \alpha\right) \Rightarrow\left(p_{i}(\alpha)=\max \left\{\gamma \in Y_{i}: \gamma \leqslant \alpha\right\}\right)(\forall \alpha \in \mathbb{Z})(\forall i \in T)$;

- с помощью равенств $\mathrm{B}_{i}(\gamma)=0\left(\forall \gamma \in Y_{i}\right)(\forall i \in M)$ аналогичной использованной алгоритмом [2] индукцией

$$
\mathrm{B}_{i}(\gamma)=\sum_{j \in \tau(i)} \min _{\beta \in U_{j}^{0}(\gamma)}\left(f_{j}(\gamma, \beta)+\mathrm{B}_{j}\left(p_{j}\left(s_{j}(\gamma, \beta)\right)\right)\right)\left(\forall \gamma \in Y_{i}\right)\left(\forall i \in T_{k} \backslash M\right)
$$

(вниз по $k)(\forall k \in[1 . . h(T)-1])$ определены функции Беллмана $\mathrm{B}_{i}$ со значениями $\mathrm{B}_{i}(\gamma) \in \mathbb{R}\left(\forall \gamma \in Y_{i}\right)(\forall i \in T)$;

- $\Psi_{i}(\alpha)=\min _{\delta \in U_{i}^{0}(\alpha)} \Phi_{i}(\alpha, \delta)\left(\forall \alpha \in\left[a_{\pi(i)}^{0} . . b_{\pi(i)}^{0}\right]\right)\left(\forall i \in T \backslash T_{0}\right)$, где $\Phi_{i}(\alpha, \beta)=$ $=f_{i}(\alpha, \beta)$ в случае $i \in M$ или $\Phi_{i}(\alpha, \beta)=f_{i}(\alpha, \beta)+\mathrm{B}_{i}\left(p_{i}\left(s_{i}(\alpha, \beta)\right)\right)$ в случае $i \notin M$; 
- по правилу

$$
\left(\beta<_{i}^{\alpha} \gamma\right) \Leftrightarrow\left(\left(\Phi_{i}(\alpha, \beta)<\Phi_{i}(\alpha, \gamma)\right) \vee\left(\left(\Phi_{i}(\alpha, \beta)=\Phi_{i}(\alpha, \gamma)\right) \wedge(\beta<\gamma)\right)\right)
$$

$$
\left(\forall \beta, \gamma \in U_{i}^{0}(\alpha)\right)
$$

во множестве $U_{i}^{0}(\alpha)$ введён порядок $<_{i}^{\alpha}\left(\forall \alpha \in\left[a_{\pi(i)}^{0} . . b_{\pi(i)}^{0}\right]\right)\left(\forall i \in T \backslash T_{0}\right)$;

- в произвольном непустом подмножестве $C \subseteq U_{i}^{0}(\alpha)$ минимальный по порядку $<_{i}^{\alpha}$ элемент обозначен $m_{i}^{\alpha}(C)\left(\forall \alpha \in\left[a_{\pi(i)}^{0} . . b_{\pi(i)}^{0}\right]\right)\left(\forall i \in T \backslash T_{0}\right)$.

Конструирование (в определенном случае оптимального) процесса даёт следующая теорема

Tеорема 2. Проиесс $(z, w) \in D^{0}$ с оценкой

$$
F(z, w)-r^{*} \leqslant F(z, w)-\sum_{i \in T_{1}} \Psi_{i}\left(a_{\pi(i)}\right),
$$

оптимальный в случае $Y_{i}=\left[a_{i}^{0} . . b_{i}^{0}\right](\forall i \in T)$, строит по обращению следующая прочедура:

ПРОЦЕДУРА first():

НАЧАЛО: положить $k=0, x_{i}=u_{i}=a_{i}\left(\forall i \in T_{0}\right)$;

СЛЕДУЮЩИЙ УРОВЕНЬ: увеличить $k$ на 1 ;

положить $u_{i}=m_{i}^{x_{\pi(i)}}\left(U_{i}^{0}\left(x_{\pi(i)}\right)\right)\left(\forall i \in T_{k}\right), x_{i}=s_{i}\left(x_{\pi(i)}, u_{i}\right)\left(\forall i \in T_{k}\right)$;

если $k<h(T)$, идти на СЛЕДУЮЩИЙ УРОВЕНЬ;

ЗАВЕРШЕНИЕ first: обозначить first () napy $(x, u)$.

3. В случае $Y_{i} \neq\left[a_{i}^{0} . . b_{i}^{0}\right](\exists i \in T)$, когда отыскание оптимального процесса не гарантировано теоремой 2, ради упрощения предполагается следующее:

$$
\begin{gathered}
j<g\left(\forall j \in T_{k-1}\right)\left(\forall g \in T_{k}\right)(\forall k \in[1 . . h(T)]) ; \\
F_{j}(x, u)=\sum_{i \in\left[\left\|T_{0}\right\|+1 . . j\right]} f_{i}\left(x_{\pi(i)}, u_{i}\right)\left(\forall j \in T \backslash T_{0}\right)\left(\forall(x, u) \in D^{0}\right) ; \\
O_{j}(x, u)=\left\{\begin{array}{l}
\sum_{g \in G_{j}} \Psi_{g}\left(x_{\pi(g)}\right) \quad \text { при } \emptyset \neq G_{j}=\left\{g \in T \backslash T_{0}: \pi(g)<j<g\right\}, \\
0 \quad \text { в остальных случаях } \\
\left(\forall j \in T \backslash T_{0}\right)\left(\forall(x, u) \in D^{0}\right) .
\end{array}\right.
\end{gathered}
$$

Следующее утверждение (теорема 3) устанавливает минимальность по определенному порядку процесса в теореме 2 и способ построения по этому порядку следующего, если возможно, процесса с пропуском построения лишь не являющихся оптимальными процессов.

ТеОрема 3. Следующие предложсения верны.

1) Процесс в теореме 2 минимален по лексикографическому порядку на $D^{0}$, вводимому правилом

$$
\begin{gathered}
((x, u) \triangleleft(z, w)) \Leftrightarrow\left(\left(u_{j}=w_{j}(\forall j \in[1 . . i-1])\right) \wedge\left(u_{i}<_{i}^{x_{\pi(i)}} w_{i}\right)\left(\exists i \in T \backslash T_{0}\right)\right) \\
\left(\forall(x, u) \in D^{0}\right)\left(\forall(z, w) \in D^{0}\right) .
\end{gathered}
$$


2) Согласно указанному порядку $\triangleleft$ следующая процедура пехt $(x, u, r)$ обращением

$$
\left(x^{2}, v^{2}\right)=\operatorname{next}\left(x^{1}, v^{1}, r^{0}\right),
$$

где $\left(x^{1}, u^{1}\right) \in D^{0}, r^{*} \leqslant r^{0}$, гарантирует выполнение условий

$$
\begin{gathered}
\left.\left(\left(\left(x^{1}, v^{1}\right) \triangleleft(y, v) \triangleleft\left(x^{2}, v^{2}\right)\right) \vee\left(\left(x^{1}, v^{1}\right)=\left(x^{2}, v^{2}\right)\right) \triangleleft(y, v)\right)\right) \Rightarrow\left(r^{0}<F(y, v)\right) \\
\left(\forall(y, v) \in D^{0}\right), \quad\left(\left(x^{1}, v^{1}\right) \triangleleft\left(x^{2}, v^{2}\right)\right) \vee\left(\left(x^{1}, v^{1}\right)=\left(x^{2}, v^{2}\right)\right) .
\end{gathered}
$$

ПРОЦЕДУРА $n \operatorname{ext}(x, u, r)$ :

НАЧАЛО: положить $(z, w)=(x, u), i=n+1$;

ПОИСК: уменъшить $i$ на 1 ; если $i \leqslant\left\|T_{0}\right\|$, идти на ЗАВЕРШЕНИЕ next;

положить $\alpha=z_{\pi(i)}, \Pi=\left\{\gamma \in U_{i}^{0}(\alpha): w_{i}<_{i}^{\alpha} \gamma\right\} ;$ если $\Pi=\emptyset$, идти на ПОИСК;

положстьв $\delta=m_{i}^{\alpha}(\Pi) ;$ если $r<F_{i}(z, w)-f_{i}\left(\alpha, w_{i}\right)+\Phi_{i}(\alpha, \delta)+O_{i}(z, w)$, идти на ПОИСК;

положить $w_{i}=\delta, z_{i}=s_{i}(\alpha, \delta)$; если $i=n$, идти на ЗАВЕРШЕНИЕ next;

УВЕЛИЧЕНИЕ: увеличить $i$ на 1 ;

положить $\alpha=z_{\pi(i)}, w_{i}=m_{i}^{\alpha}\left(U_{i}^{0}(\alpha)\right), z_{i}=s_{i}\left(\alpha, w_{i}\right) ;$ если $i<n$, идти на УВЕЛИЧЕНИЕ;

ЗАВЕРШЕНИЕ next: обозначить next $(x, u, r)$ napy $(z, w)$.

4. В общем случае получение оптимального процесса даёт следующая теорема.

Теорема 4. Оптимальный процесс $\left(x^{*}, u^{*}\right)$ и оптимальное значение $r^{*}$ находит при помощи first() и нескольких обращений $\kappa$ nроцедуре nеxt $(x, u, r)$ следующий итеращионный алгоритм.

\section{АЛГОРИТМ:}

НАЧАЛО: положить $\left(x^{\prime}, u^{\prime}\right)=\operatorname{first}(), r^{\prime \prime}=\infty$;

ИЗМЕНЕНИЕ: положить $(x, u)=\left(x^{\prime}, u^{\prime}\right)$;

ИТЕРАЦИЯ: положить $r=F(x, u)$; если $r<r^{\prime \prime}$, положить $\left(x^{\prime \prime}, u^{\prime \prime}\right)=$ $=(x, u), r^{\prime \prime}=r$;

положить $\left(x^{\prime}, u^{\prime}\right)=\operatorname{next}\left(x, u, r^{\prime \prime}\right) ;$ если $(x, u) \neq\left(x^{\prime}, u^{\prime}\right)$, идти на ИЗМЕНЕНИЕ;

ЗАВЕРШЕНИЕ АЛГОРИТМА: положить $\left(x^{*}, u^{*}\right)=\left(x^{\prime \prime}, u^{\prime \prime}\right), r^{*}=r^{\prime \prime}$; остановиться.

\section{СПИСОК ЛИТЕРАТУРЫ/ REFERENCES}

1. В. А. Емеличев, В. Г. Овчинников, "Применение метода построения последовательности планов к решению задачи обустройства нефтяных месторождений" // Докл. АН БССР, 1982. T. 26, № 4. C. 344-347. [V. A. Emelichev, V. G. Ovchinnikov, "Application of the method of constructing successive plans to the problem of oil field equipping", Dokl. Akad. Nauk BSSR, 1982, vol. 26, no. 4, pp. 344-347. (In Russian)].

2. В. Г. Овчинников, “Алгоритмы динамического программирования оптимальных и близких к ним процессов" / Труды пятой Всероссийской конферениии с международным участием (29-31 мая 2008 г.). Часть 4, Информационные технологии в математическом моделировании / Матем. моделирование и краев. задачи, Самара: СамГТУ, 2008. С. $107-$ 112. [V. G. Ovchinnikov, "Algorithms of dynamic programming for optimal and similar 
processes", Proceedings of the Fifth All-Russian Scientific Conference with international participation (29-31 May 2008). Part 4, Matem. Mod. Kraev. Zadachi, Samara, Samara State Technical Univ., 2008, pp. 107-112. (In Russian)].

3. В. Г. Овчинников, "К алгоритмам динамического программирования оптимальных процессов"// Вестн. Сам. гос. техн. ун-та. Сер. Физ.-мат. науки, 2012. №3(28). C. 215-218. doi: 10.14498/vsgtu1102. [V. G. Ovchinnikov, "On the algorithms of dynamic programming for optimal processes", Vestn. Samar. Gos. Tekhn. Univ. Ser. Fiz.-Mat. Nauki, 2012, no. 3(28), pp. 215-218. (In Russian)].

Поступила в редакцию 01/IX/2013;

в окончательном варианте - 13/XII/2013;

принята в печать - $17 / \mathrm{I} / 2014$.

MSC: 90B10; 90C90, 65K05

\section{ON THE DYNAMIC PROGRAMMING ALGORITHM UNDER THE ASSUMPTION OF MONOTONICITY}

\section{G. Ovchinnikov}

Samara State Technical University,

244, Molodogvardeyskaya st., Samara, 443100, Russian Federation.

We formulate a discrete optimal control problem, which has not been considered earlier, which arises in the design of oil and gas networks. For this problem we set four theorems so that you can have a process, the optimal process and the optimum value. Necessary and sufficient conditions we give in Theorem 1. Under these conditions, by Theorem 1, we get not empty attainability intervals. For each interval, we choose the grid-a subset of its points, where by an arbitrary point of interval, we find the nearest point on the left. By means of such approximations, we define the Bellman functions on the grids. Using Bellman functions in Theorem 2 we give the process and we evaluate its deviation from the optimal process. In Theorem 2, we guarantee, that the given process is optimal when the attainability intervals and their grids coincide. In other cases, to get the optimal process, we use Theorem 3 and Theorem 4. In Theorem 3 we set that the process given in Theorem 2, is minimal in the lexicographical order which we introduce using Bellman functions. In Theorem 3 we give procedure that builds, if possible, in this order, the next process, skipping only the processes that are not optimal. We find the optimal process and the optimal value by Theorem 4, starting from the process given in Theorem 2, using one or more calls of the procedure given in Theorem 3.

Keywords: dynamic programming, Bellman functions, procedures, algorithms.

Received 01/IX/2013;

received in revised form $13 / \mathrm{XII} / 2013$;

accepted $17 / \mathrm{I} / 2014$.

ISSN: 2310-7081 (online), 1991-8615 (print); doi: http://dx.doi.org/10.14498/vsgtu1257 (C) 2014 Samara State Technical University.

Citation: V. G. Ovchinnikov, "On the Dynamic Programming Algorithm under the Assumption of Monotonicity", Vestn. Samar. Gos. Tekhn. Univ., Ser. Fiz.-Mat. Nauki [J. Samara State Tech. Univ., Ser. Phys. \& Math. Sci.], 2014, no. 1(34), pp. 186-191. doi: 10.14498/vsgtu1257. (In Russian)

Author Details: Valery G. Ovchinnikov, Senior Lecturer, Dept. of Oil and Gas Fields Development.

E-mail address: ovchinnikov42@mail.ru 